\title{
Stress-Strain State Analysis and Fatigue Prediction of D16T Alloy in the Stress Concentration Zone Under Combined Tension-Torsion Load
}

\author{
Isaac SOLOMON*, Evaldas NARVYDAS**, Gintautas DUNDULIS*** \\ *Independent Researcher, 11 Erran Street, Purasawalkam 600007, Chennai, India, E-mail: shmuelisaac@gmail.com \\ **Kaunas University of Technology Studentu g. 56, 51424 Kaunas, Lithuania, E-mail: evaldas.narvydas@ktu.lt \\ ***Kaunas University of Technology Studentu g. 56, 51424 Kaunas, Lithuania, E-mail: gintautas.dundulis@ktu.lt \\ cross ref http://dx.doi.org/10.5755/j02.mech.28454
}

\section{Introduction}

Engineering machines and components are prone to structural failures during their service time due to certain technical reasons. The technical breakdowns sometime lead to high economic imbalance and can also be fatal to life and property. The unfortunate failures are often due to some flawed engineering manufacturing techniques or even due to material failure [1].

Predicting the failure and evaluating the breakage characteristics of engineering components are crucial in determining the life of the component and also increase their maintenance and safety in daily life. The fundamental necessity is to analyse these structural failures with a hope of obtaining a certain set of results which might be helpful in the improvements of the structural integrity of the components. The analysing and predictions of material failures can be attained through experimental and numerical simulations in tandem.

This research study deals with the modelling and numerical simulations of an aluminium alloy specimen in $3 \mathrm{D}$ stress-state and thereby predicting the fatigue failure of the material subjected to external cyclic loadings. To predict the failure of a component, a specimen with an induced hole can be evaluated through cyclic loading process. It is based on the fact that the presence of a holes tends to modify the stresses present locally on the component that the elastic deformation and the stresses attributed with them are totally insufficient for the design against fracture. It is based on the assumption that the specimen undergoes complete fracture when the hole reaches its critical size even though the stress at the critical area is much lower than the yield stress of the component. The critical size of the hole is based on the application of the load and the number of load cycles it undergoes. [2].

The main aim of this research is to present and validate the numerical method for the study of the influence of holes present in the engineering components. Finite element method was applied for numerical simulation. In this study the tension, torsion, combined tension-torsion and fatigue loads was applied. The experimental testing data of mechanical properties was used in numerical simulation as input data. This research study investigates the three-dimensional stress-strain state and fatigue prediction of D16T aluminium alloy which is predominantly used in the aerospace and automobile industries for their high strength-to-weight ratio and much better physical properties. The different specimen models are then analysed and the most efficient one was selected for the preliminary experimental tests.

\section{Experimental testing of Aluminium alloy}

\subsection{Material}

The material which is considered in this research study is D16T GOST 4784-97 aluminium alloy which is equivalent to the Al-alloy EN AW-2024. This material is a modified version of duralumin alloy D16 and can also be modified to certain specific dimensions according to the applications. The material D16T are predominantly used in the aerospace and mechanical industries and its applications vary from miniature technical components to large scaleequipment. The material D16T is produced by modifying certain parameters of duralumin which consists of $90 \%$ of pure $\mathrm{Al}, 4 \%$ of $\mathrm{Cu}, 1 \%$ of $\mathrm{Mg}$ and $0.5 \%$ to $1 \% \mathrm{Mn}$. The temperature of the duralumin alloy increased to about $500^{\circ}$ $\mathrm{C}$ in the manufacturing process, followed by immediate quenching in water. The mechanical properties are presented in Table 1.

Table 1

Mechanical Properties of D16T Al-alloy [3]

\begin{tabular}{|c|c|}
\hline Mechanical Property & Data \\
\hline Yield Strength, MPa & 320 \\
\hline Ultimate Strength, MPa & 530 \\
\hline Density, kg/m & 3770 \\
\hline Young's Modulus, MPa & 70000 \\
\hline Elongation percentage, $\%$ & $10.5-10.9$ \\
\hline Brinell Hardness, - & 144 \\
\hline
\end{tabular}

2.2. Test specimen

Tensile tests were performed according to ASTM E8/E8M standard recommendations. $4 \mathrm{~mm}$ diameter specimens were machined for the tensile test. Three specimens were produced from D16T Al-alloy for the tensile tests. The specimens were produced according to the type of testing employed on them. The tensile test specimen shown in Fig. 1 has a solid cylindrical structure [3].

\subsection{Test specifications}

The tensile tests are done on the Tinius Olsen H25KT testing machine in the Mechanics' Laboratory of the Faculty of Mechanical Engineering and Design, Kaunas University of Technology, Kaunas, Lithuania. The Tinius Olsen H25KT is a Universal Testing Machine (UTM) with a load capacity of $25-\mathrm{kN}$.

Three of the test specimens from D16T Al-Alloy (Fig. 1, b) were experimentally tested in the Tinius Olsen 
H25KT strength testing machine using a quasi-static uniaxial tension method. Each of these specimens was tested with uniform parameters one after another to achieve maximum linear results.

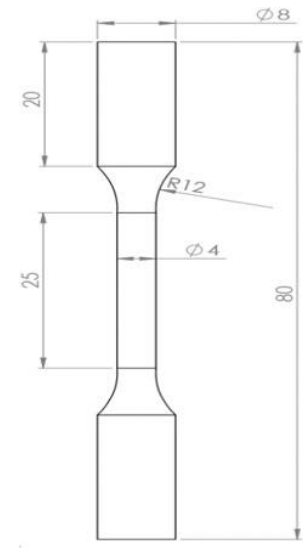

a

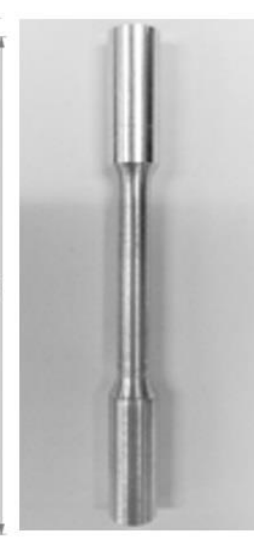

b
Fig. 1 Schematic drawing: a) laboratory produced; b) specimen for the experimental tensile test

The specimen was then loaded with a uniaxial quasi-static tensile force at a displacement-controlled rate of $1.5 \mathrm{~mm} / \mathrm{min}$. The constant displacement-controlled loading was achieved with the help of a servo motor until the failure of the component through fracture occurs. The displacement-controlled loading of $1.5 \mathrm{~mm} / \mathrm{min}$ was taken as an arbitrary value suitable for testing tensile specimens. Imperative parameters of the tension test are presented in Table 2.

Table 2

Imperative parameters of the tension test

\begin{tabular}{|c|c|}
\hline Property & Specifications \\
\hline Test method & Uniaxial quasi-static tension \\
\hline Loading type & $\begin{array}{c}\text { Displacement-controlled } \\
(1.5 \mathrm{~mm} / \mathrm{min})\end{array}$ \\
\hline Duration of the load & Until breakage \\
\hline Specimen length (total) & $80 \mathrm{~mm}$ \\
\hline Specimen length (testing region) & $25 \mathrm{~mm}$ \\
\hline Specimen diameter (at the grips) & $8 \mathrm{~mm}$ \\
\hline $\begin{array}{c}\text { Specimen diameter (testing re- } \\
\text { gion) }\end{array}$ & $4 \mathrm{~mm}$ \\
\hline
\end{tabular}

The lateral and longitudinal deformation of the specimen through the application of the tensile load was measured optically by a camera pointing straight at the testing zone and the various displacement values were presented in the system inbuilt software through optical extensometers with reference to the markings on the specimen.

The above Fig. 2 is a photograph of the mounted specimen before loading captured by the GigE Cam port 1 (17 frames per second) inbuilt in the Tinius Olsen machine. The Fig. 3 shows the various measurement tracking of the extensometer gage length corresponding to the markings made on the specimen.

The displacements which occur due to the tensile loading are measured using the tracker employed by the extensometer strain gages which measure the longitudinal displacements of the specimen. The extensometer tracker is manually placed on the markings made on the specimen and will measure the displacements corresponding to the markings. A total of nine sets of extensometer markings were made on the specimen just to ensure maximum accuracy for the measurements

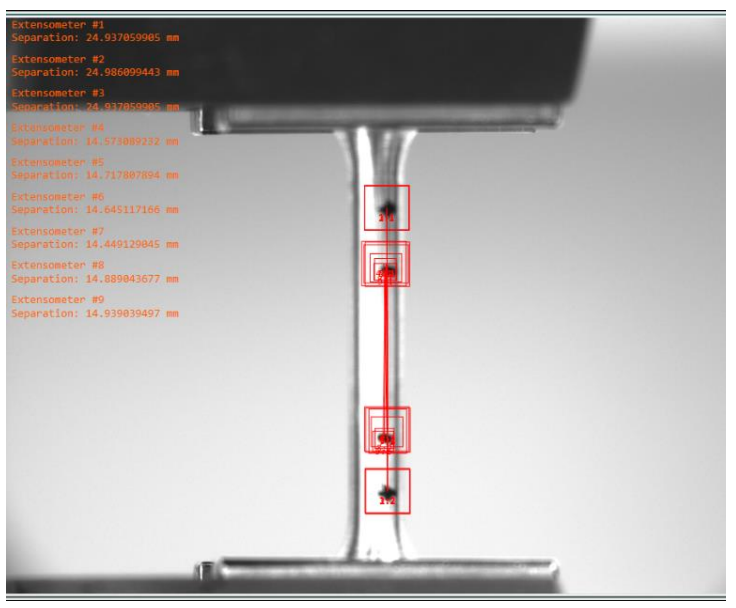

Fig. 2 Extensometer references on the specimen

The loading would stop immediately once the specimen reached its final and critical point of fracture. This critical loading point was stored in the system software and the different values corresponding to the engineering stress/strain parameters were obtained. The various results corresponding to the tensile tests of the three specimens were obtained from the system inbuilt software.

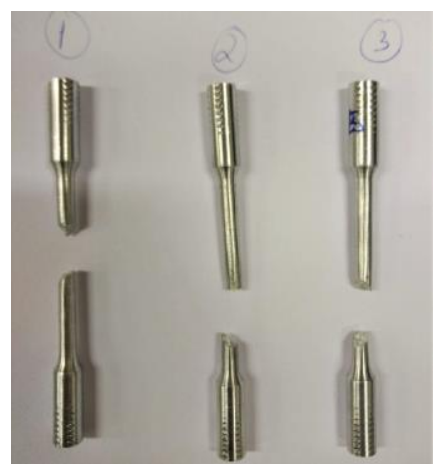

Fig. 3 Fractured specimens after batch experimentation

The above Fig. 3 is a photograph of all the three fractured specimens through the tensile load until breakage. All the three specimens were loaded uniformly with a quasistatic uniaxial tensile load at room temperature and the all three recorded almost similar values of maximum force and ultimate stress.

\subsection{Experimental results}

The various results like stresses, strain \%, displacements and forces can be obtained by the software inbuilt in the Tinius Olsen testing machine. The testing results are presented in Table 4 . The values obtained from the testing machine are the values that relate to the Engineering stressstrain curves and these values are converted to True stressstrain data for further implementation in the numerical study.

The conversion from the Engineering stress-strain data to the True stress-strain data was of major importance since the Engineering stress-strain data assumes that the cross-section of the given specimen was a fixed variable. The True stress-strain data takes into account the implicit change in the cross-section of the specimen at different 
stages of the test, hence the data obtained from this curve serves as central information for the input in the numerical study.

Table 4

Imperative results of the experiment

\begin{tabular}{|c|c|c|c|}
\hline Variable & Specimen 1 & Specimen 2 & Specimen 3 \\
\hline Maximum force, N & 8830 & 8900 & 8520 \\
\hline Ultimate stress, MPa & 450 & 453 & 434 \\
\hline Break-distance, $\mathrm{mm}$ & 9.31 & 9.32 & 8.96 \\
\hline
\end{tabular}

The values of the Engineering strain are converted to True strain data by using the formula:

$$
\begin{aligned}
& \varepsilon_{T}=\int \frac{d_{L}}{L}=\ln \left(\frac{L}{L_{0}}\right), \\
& \varepsilon_{T}=\ln \left(\frac{L_{0}+\Delta L}{L_{0}}\right), \\
& \varepsilon_{T}=\ln \left(1+\varepsilon_{E}\right),
\end{aligned}
$$

where: $L_{0}$ is initial length; $\Delta L$ is change in length.

The values of the Engineering stress are then converted to True stress values depending on the values of the true strain data.

$$
\sigma_{T}=\operatorname{EXP}\left(\varepsilon_{T}\right) \sigma_{E},
$$

where: $\sigma_{T}$ is the true stress; $\varepsilon_{T}$ is the true strain; $\sigma_{E}$ is the engineering stress [12].

Hence the values of True stress and True strain for these specimens are plotted against each other for determining the yield point of the material under quasi-static uniaxial tensile load.

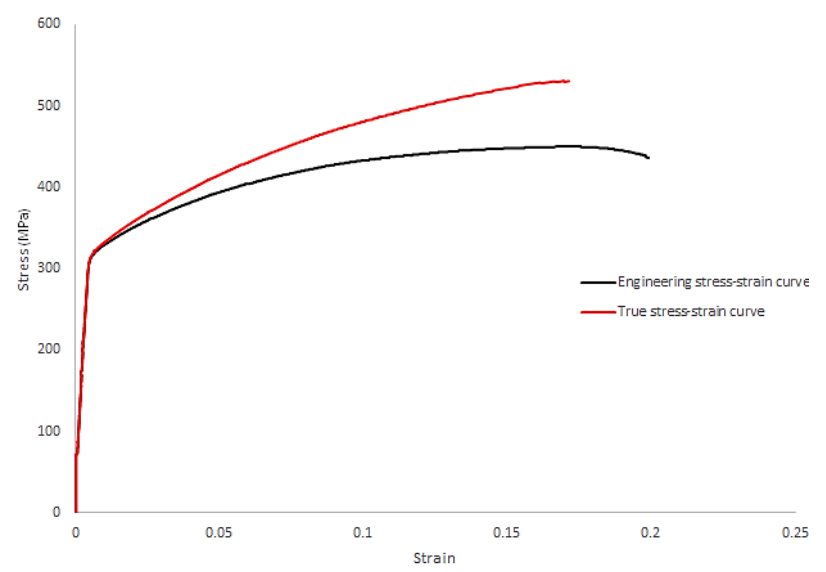

Fig. 4 Stress-strain plot of specimen

The above Fig. 4 is the plot of the Stress against Strain for Specimen 1. This plot also gives a comparison of the Engineering stress-strain plot and the True stress-strain plot. The two curves almost seem equivalent in the elastic region due to the quasi-static type of loading while they deviate after the material starts yielding, and this non-equivalency is characterized by the strain rate after the yield point [5].
It is of substantial consideration that the yield stress of the material (D16T) is to be calculated from these plots so as to input the data into the numerical study, hence the evaluation of the yield stress of the material is done with the help of rudimentary engineering techniques.

2.5. Evaluating the yield stress of the material based on the experimental results

The fundamental procedure used in the evaluating of the yield stress of the material is the implementation of the $0.2 \%$ offset proof stress on the plot.

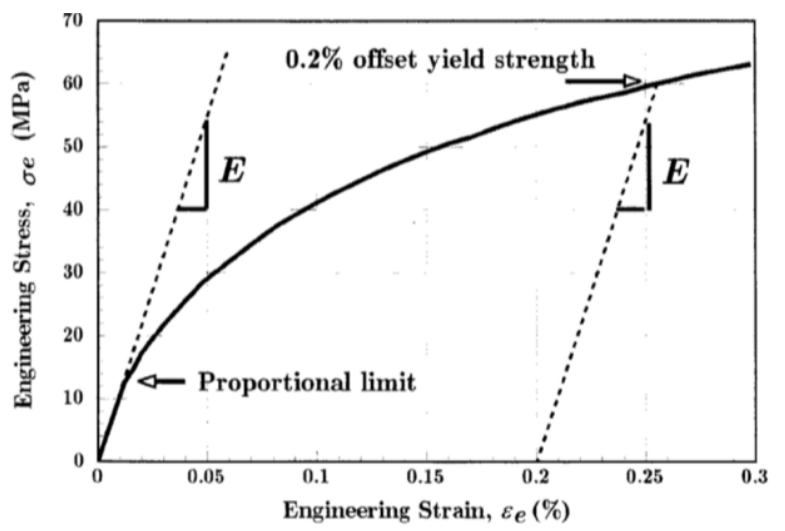

Fig. 5 Proposed evaluation plot of yield stress [2]

The Fig. 5 depicts the method for determining the yield stress by using the $0.2 \%$ offset approach which utilises a parallel line to the stress-strain curve.

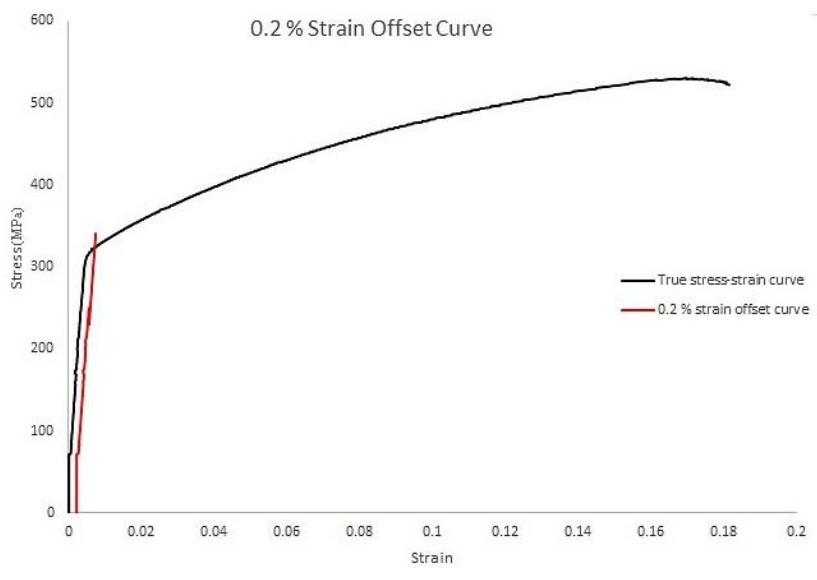

Fig. $60.2 \%$ offset proof stress

The Fig. 6 is a plot of True stress against True strain along with the $0.2 \%$ offset proof stress. The $0.2 \%$ proof stress is the quantity of stress that results from $0.2 \%$ of plastic strain of the material under tension.

This method is undertaken since the stress point at which the material transits from elastic state to plastic state is not vivid. This method utilizes the construction of another parallel line offset to the elastic region by $0.002 \mathrm{~mm} / \mathrm{mm}$ or $0.2 \%$. The yield stress can then be evaluated by the intersecting point of the offset line with the stress-strain curve. In this case, the offset line is set to intersect the True stressstrain curve at approximately $320 \mathrm{MPa}$. Albeit, from the plot, it is evident that the maximum ultimate stress is approximately $530 \mathrm{MPa}[6]$. 


\section{Computational results}

The numerical simulations were carried out to simulate the mechanical behaviour of the experimental models under quasi-static uniaxial tensile load. A three-dimensional constitutive model was developed using the CAD software SOLIDWORKS ${ }^{\circledR}$. The three-dimensional geometries were constructed using the data based on the experimental setup and were up to the scale. The constitutive model was then solved for Static Non-linear conditions taking into consideration the results of the experimental tests. The finite element modelling of the geometry was intricately done using physics-based meshing techniques for accurate results. The boundary conditions of the model were similar to the loads and constraints are given to the experimental specimens.

The finite element method approach was carried out on the tubular specimen with a $2 \mathrm{~mm}$ central hole in the prospect of investigating the stress direction on hollow cylindrical structures of D16T Al-alloys. The model prepared for analysis is presented in Fig. 7, a. The von - Mises stress criterion was observed for the specimen under three different loading conditions, tension, torsion and combined load; and the stress variables were observed at three distinct points -Initial load (a), at yield load (b) and at the fracture point (c).

The mechanical properties of the material (D16T Al-alloy) used in the numerical simulations are input in the SOLIDWORKS material library as a custom material and the various such properties are determined in the experimental results.

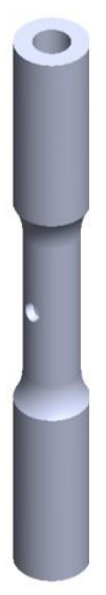

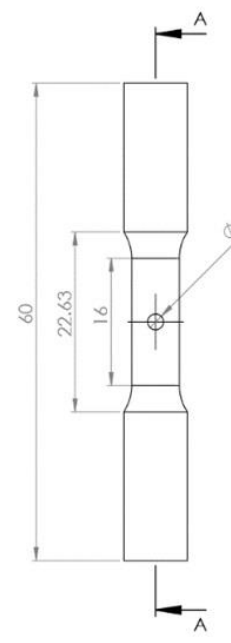

b

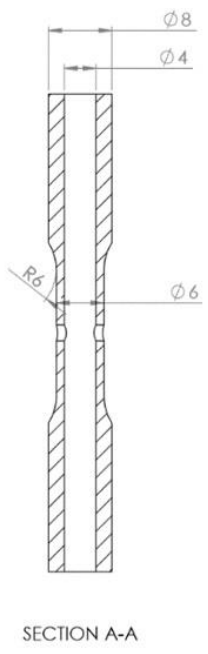

c
Fig. 7 Isometric view (a); schematic diagram (b); section view (c) of the specimen used in the numerical simulations

The material stress-strain data for the static nonlinear numerical study is assigned to the simulation model and the value starts with the yield limit of the material under tension and goes on till the ultimate stress so as to generate a Non-linear model for the simulation. The Finite Element Model was created using physics-based meshing techniques and the meshing was increased in the areas of major concentration. The shape of the finite elements used in this simulation was tetrahedral shaped elements with a mid - node capability to increase the accuracy of the solution. The type of constraint used in this numerical study is a fixed support constraint which is a constant for all the three types of loading conditions. The fixed support constraint is applied on the lower face of the model thereby, constraining all the degrees of freedom of the lower part of the component.

The numerical study is solved for static Non - linear conditions for all the three types of loadings: a) tension, b) torsion and c) combined loads, since major importance is given for the behaviour of the material after the yielding point. The non - linear material data is obtained from the results of the experiments under tensile loads. The time steps of the study are kept constantly at 1 second and the time increments are kept at a fixed rate of 0.075 seconds. The numerical study is solved in large displacement mode along with the large strain mode. The type of solver used in the numerical simulations are FFEPlus solver which employs an iterative approximation technique by assuming a solution and then iterating the errors until the solution converges with the accepted errors. Owing to its relatively low memory usage and use of iterative approximation methods, the numerical simulation is solved using FFEPlus solver over other direct solving techniques.

\subsection{Results of the tensile study}

The tubular specimen with a $2 \mathrm{~mm}$ central hole is solved for uniaxial static non - linear conditions for a tensile load of $5000 \mathrm{~N}$ applied on the top face of the specimen. This force of $5000 \mathrm{~N}$ is gradually applied to the model in steps of 13 increments and the load increases in increments until failure. An initial load of $5000 \mathrm{~N}$ is applied on to specimen for the tension study. The von - Mises stress criterion was observed for the applied tensile loads at three distinct stress points: (a) initial loading, (b) when the material starts to yield and (c) at the fracture point and are presented in Fig. 8.

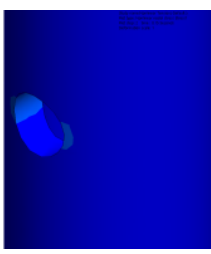

a

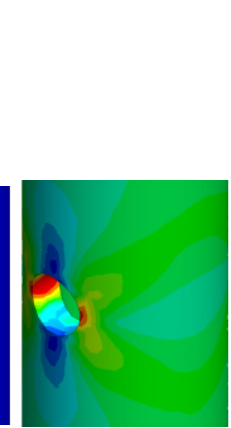

b

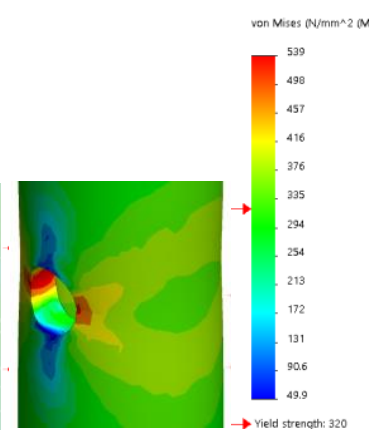

$\mathrm{c}$
Fig. 8 Equivalent von-Mises stress contours at the hole at three distinct points for the tensile loading, initial loading (a), when the material starts to yield (b) and at the fracture point (c)

The change in the shape of the model prior to the loading conditions adheres to the experimental results of the specimen under tension and the elliptical distribution of the von - Mises stress contours along the circumference of the $2 \mathrm{~mm}$ central hole for tensile load evidently obeying the general elasticity theory [5].

The results of the static uniaxial tensile Non-linear numerical study versus von-Mises stress are presented in Table 6.

It is evident from these values that the transition of the material from the elastic to elastic-plastic region and elastic-plastic to completely plastic region is continuous, 
there was a sudden decline of the structural integrity of the model and an enormous increase in the stress-state due to the presence of the $2 \mathrm{~mm}$ hole; the prediction of the failure can be assessed by evaluating the direction of the stress contour along the sides of the hole [7, 8].

Table 6

Results of the static uniaxial tensile non-linear numerical study

\begin{tabular}{|c|c|}
\hline Load, $N$ & von-Mises stress, MPa \\
\hline 375 & 75.3 \\
\hline 750 & 151 \\
\hline 1125 & 226 \\
\hline 1500 & 301 \\
\hline 1875 & 317 \\
\hline 2250 & 324 \\
\hline 2625 & 332 \\
\hline 3000 & 342 \\
\hline 3375 & 351 \\
\hline 3750 & 363 \\
\hline 4125 & 420 \\
\hline 4500 & 485 \\
\hline 4875 & 539 \\
\hline
\end{tabular}

The Fig. 9 gives a clear understanding of the change of stress - state with incremental loadings in a tensile simulation owing to a slow rate of deformation at the yield stage and a growing rise in stress due to the presence of notches (holes) which contributes to a major portion of the failure of the model at higher loads. At the point where the material starts to yield, the steady-slow rate of change of von-Mises stress with increasing load is characterized by the distribution of stress around the stress concentration zone where the increase in stress value is almost constant owing to the behaviour of plastic yielding.

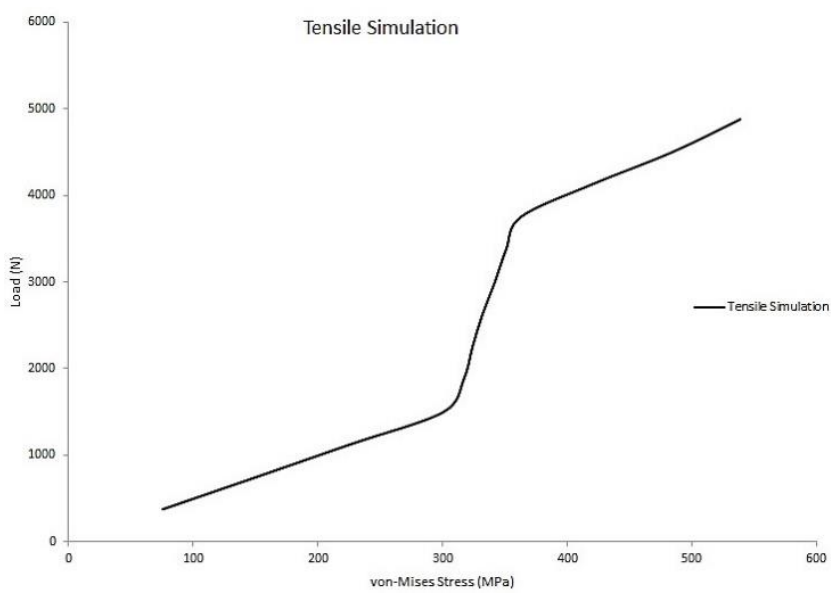

Fig. 9 Load step vs Equivalent von-Mises stress at the hole plot from tensile simulation

\subsection{Results of the torsional study}

The tubular specimen with a $2 \mathrm{~mm}$ central hole is solved for static non - linear conditions for a torsional load of $8 \mathrm{Nm}$ in the clockwise direction applied on the top face of the specimen. This moment of $8 \mathrm{Nm}$ is gradually applied to the model in steps of 13 increments (as explained in the paragraph 3.1).

The Fig. 10 gives a depiction of the non-linear stress distribution contours due to an incremental torsional load of $8 \mathrm{Nm}$ in the clockwise direction leading to the sudden twisting of the area around the central hole with an increase in the load after the yield point of the material. In Table 7 the results of the static torsional non - linear numerical study at different load steps is presented.

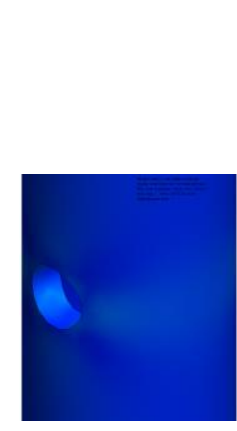

a

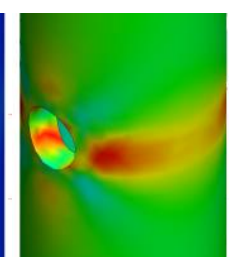

b

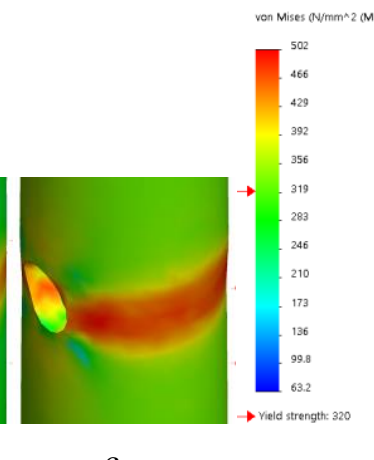

c
Fig. 10 Equivalent von-Mises stress contours at the hole at three distinct points for torsional loading, initial loading (a), when the material starts to yield (b) and at the fracture point (c)

From the Table 7 values, it is obvious that the rise in the strain rate is rapid after the material's yielding point, which adds up to the decline of the structural strength under such loading conditions in the presence of a crack, where the stresses produced in the plasticity zone are much more than those produced before the yield point. The change in the size and shape of the component under torsional loads are acceptable and obeys the fundamental laws of elasticity and crack propagation theory $[2,9]$.

Table 7

Results of the static torsional non- linear numerical study

\begin{tabular}{|c|c|}
\hline Load, Nm & von-Mises stress, MPa \\
\hline 0.615 & 65.7 \\
\hline 1.23 & 131 \\
\hline 1.845 & 197 \\
\hline 2.46 & 263 \\
\hline 3.075 & 311 \\
\hline 3.69 & 321 \\
\hline 4.305 & 326 \\
\hline 4.92 & 333 \\
\hline 5.535 & 349 \\
\hline 6.15 & 391 \\
\hline 6.765 & 430 \\
\hline 7.38 & 469 \\
\hline 7.995 & 502 \\
\hline
\end{tabular}

The Fig. 11 shows the plot of load against stress where we can see a steady exponential rise in the stress state up to the yield point of the material and an unstable rise at the plastic zone.

\subsection{Results of the combined tension-torsion study}

The tubular specimen with a $2 \mathrm{~mm}$ central hole is solved for static non-linear conditions for both tensile load of $5000 \mathrm{~N}$ and torsional load of $8 \mathrm{Nm}$ in the clockwise direction applied on the top face of the specimen. This combined force is gradually applied to the model in steps of 13 increments. 


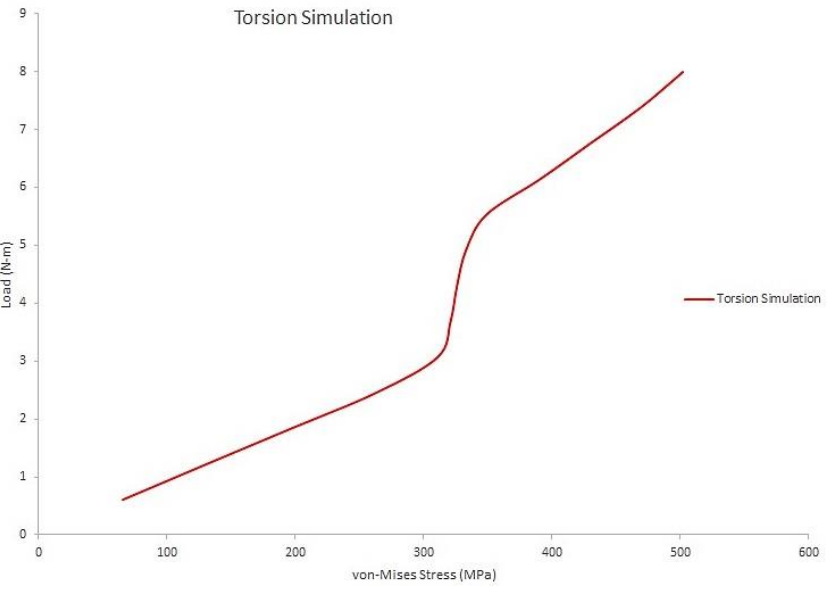

Fig. 11 Load step vs equivalent von-Mises stress at the hole plot from torsional simulation

The resultant stress values obtained from the simulation employing combined loading provides a different evaluation of the stress shape and direction in Fig. 12. It is seen that the elliptical stress contours occurring due to the presence of the tensile force gets carried away with the twist angle due to torsion.

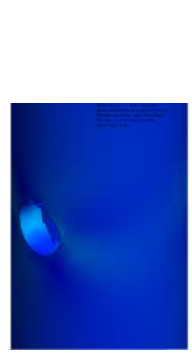

a

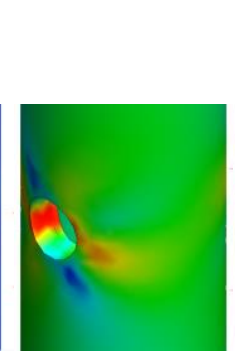

b

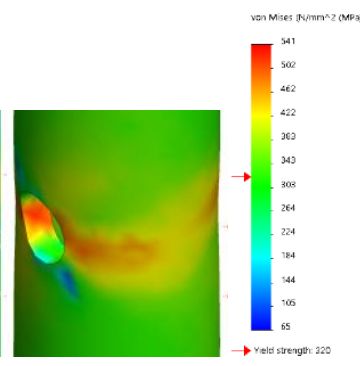

c
Fig. 12 Equivalent von-Mises stress contours at the hole at three distinct points for the combined tension-torsion loading, initial loading (a), when the material starts to yield (b) and at the fracture point (c)

Table 8

Results of the static torsional non - linear numerical study

\begin{tabular}{|c|c|c|}
\hline Load, Nm & Load, N & von-Mises stress, MPa \\
\hline 0.62 & 375 & 101 \\
\hline 1.23 & 750 & 201 \\
\hline 1.85 & 1125 & 292 \\
\hline 2.46 & 1500 & 322 \\
\hline 3.08 & 1875 & 334 \\
\hline 3.69 & 2250 & 350 \\
\hline 4.31 & 2625 & 378 \\
\hline 4.92 & 3000 & 486 \\
\hline 5.54 & 3375 & 541 \\
\hline 6.15 & 3750 & 577 \\
\hline
\end{tabular}

The axial load of $5000 \mathrm{~N}$ is applied to the model in order to decrease the condition of hole closure and it is to be noted that, from the Fig. 13, the resultant stresses which corresponds to the application of the axial load is much smaller than that of the torsional load because of the shearing nature of the torsional load [10]. One can also note that the presence of "neck" occurs early when compared with the conventional single-acting loads. The neck region is prone to excessive deformations with the application of tensile and torsional loads in tandem and the direction of the stress is seen making an angle with the torsional angle of twist. The results of the static torsional Non - linear numerical study at different torsional load steps is presented in Table 8.

In the combined loading study, the contour point at which the load sections are separated are assumed to be the critical load points of the tensile and torsion loads. This study is done to reveal the influence of non-proportional loading (tensile and torsional) for cylindrical (axisymmetric) components to predict the stress direction and propagation [11].

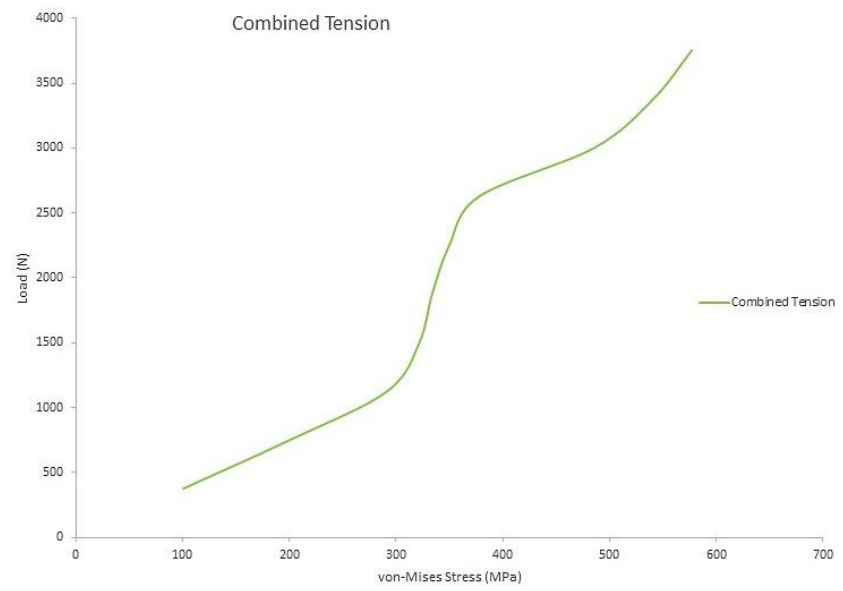

a

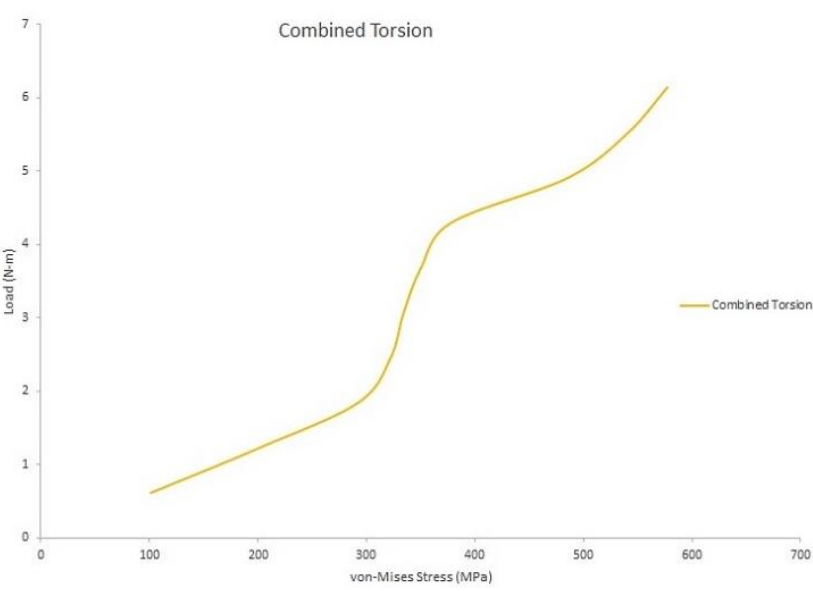

b

Fig. 13 Plots of tension load vs equivalent von-Mises-stress at the hole region (a), torsional load vs equivalent von-Mises stress at the hole region (b) for the combined tension-torsion loading study

\subsection{Results of the combined load fatigue study}

The fatigue simulation for the hollow specimen was done in SOLIDWORKS by utilising its implicit method of solving linear and non-linear fatigue damage. The same input load data which was given in the combined-load simulations was set as the boundary conditions for the fatigue simulations. The tensile load steps until $4000 \mathrm{~N}$ and torsional load steps until $7 \mathrm{Nm}$ are given from the combined loading setup as the input for the fatigue study for evaluating the fatigue life and damage. The alternating stress is computed using the equivalent von-Mises stress and the mean stress correction uses the Goodman method.

The Fig. 14 is the representation of the fatigue S-N curve (the Wohler curve) for the material D16T Al-alloy in- 
corporated in the SOLIDWORKS materials library. The values of these curves are based on experimental data and each of those stress amplitude values corresponding to the number of applied load cycles are presented in the Table 9. The stress amplitude of $294 \mathrm{MPa}$ for 1E3 cycles with a constant amplitude ( $L R=0)$ loading ratio is simulated.

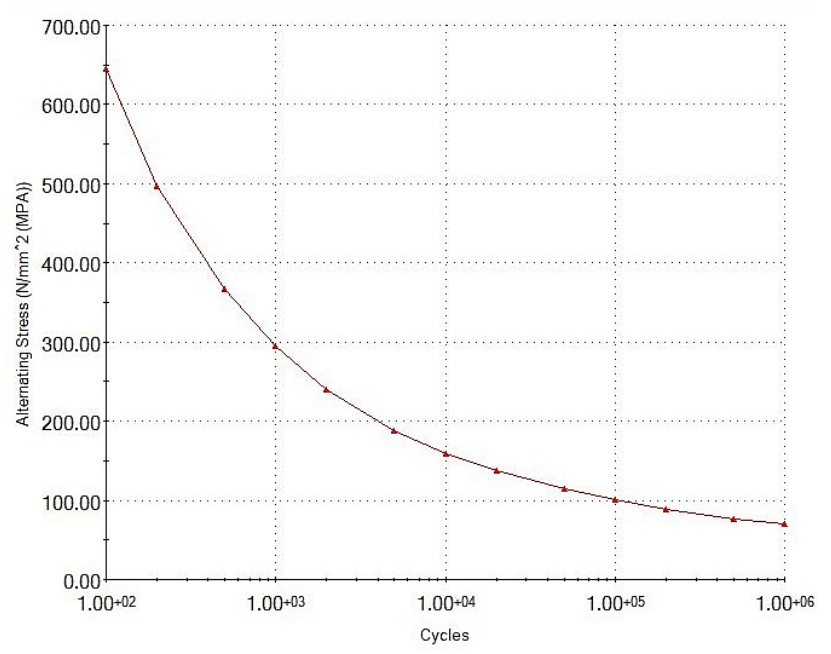

Fig. 14 S-N curve of D16T Al-alloy

Fatigue S-N curve data

Table 9

\begin{tabular}{|c|c|}
\hline No. of Cycles & Stress amplitude, MPa \\
\hline 100 & 645.6 \\
\hline 200 & 497.2 \\
\hline 500 & 366.1 \\
\hline 1000 & 294.3 \\
\hline 2000 & 239.9 \\
\hline 5000 & 188.0 \\
\hline 10000 & 158.3 \\
\hline 20000 & 137.3 \\
\hline 50000 & 114.5 \\
\hline 100000 & 100.9 \\
\hline 200000 & 88.8 \\
\hline 500000 & 76.7 \\
\hline 1000000 & 70.0 \\
\hline
\end{tabular}

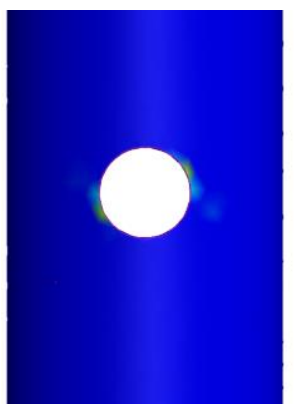

a
Damage Percentage

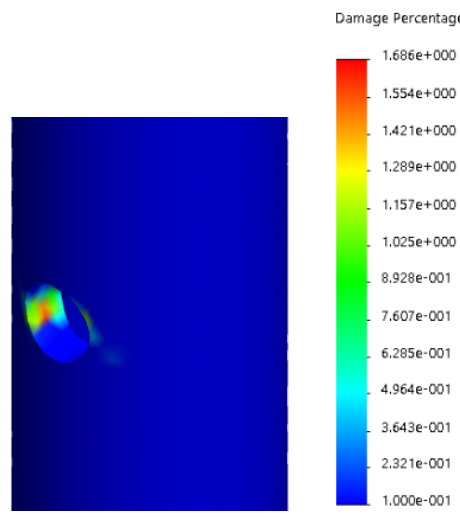

b
Fig. 15 Damage percentage contour normal view (a), isometric view (b)

The Fig. 15 illustrates the fatigue damage area in the stress concentrators which are given in the overall percentage for the combined tension-torsion load for a load cycle of 1E3 cycles. The maximum damage areas are clearly visible which are inclined at an angle to the lateral plane of the stress concentrators due to the effect of torsional and tensile loads. The fatigue study is done in order to present the vulnerable regions in the stress concentrators which might predominantly affect the component leading to its failure in lower cycles ( $1 \mathrm{E} 3$ cycles) of stress amplitudes.

The Fig. 16 shows the contour of fatigue life around the stress concentrator and it can be observed that the fatigue life at the hole is $5.9 \mathrm{e}^{4}$ cycles due to the intensity of stress acting at an angle to the lateral hole region due to the combined action of torsion and tension.

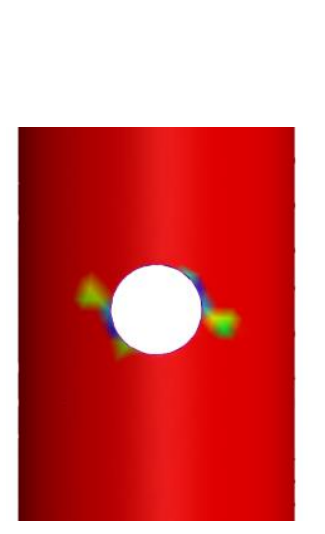

a

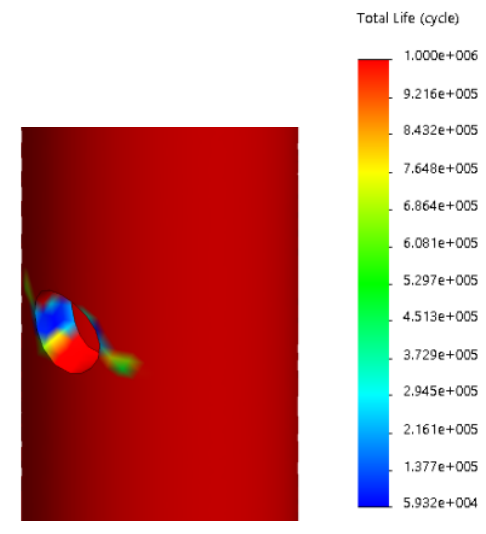

b
Fig. 16 Fatigue life contour normal view (a), isometric view (b)

\section{Conclusions}

The research studied the influence of holes present in the engineering components and an experimental study was carried out which was then attempted against numerical simulations. The tensile test was implemented for the experimental tests and the resultant material properties of the D16T alloy was evaluated and translated into data which are the fundamental inputs for attempting the FEM. The finite element models were created and the various simulations like tension, torsion, combined tension-torsion and fatigue tests were simulated with the hollow cylindrical model and the distinct stress-strain contours are evaluated in the stress concentrators at three distinct points of loading (initial loading, at the yield point and at the fracture point). The fatigue damage contours and the vulnerability in the stress concentrator regions for the fatigue life are presented with for $1 \mathrm{e}^{+3}$ cycles of constant amplitude loading.

For all the three different type of loadings, it is observed that the maximum stress and failure direction occurs in the plane of the principal stress corresponding to the different modes (I, II and III) of failure for Al-Alloy D16T which has a partial brittle nature and the stress-strain results of the material under these loading modes are presented. This research study provides necessary information of the material characteristics by approximating its load-deformation behaviour for lab-scale tension, torsion, combined and fatigue loads thereby, serving as an initial tool in determining the failure prognosis of D16T. Due to the limited resources of the experimentation, the research study limits itself to the tensile experiments but provides necessary data for future experiments on LCF and HCF fatigue tests for an in depth analysis and failure predictions of D16T aluminium alloy. 


\section{References}

1. Richard, H.; Fulland. M.; Sander M.; Kullmer, G.; Examples of Fatigue Crack Growth in Real Structures. https://www.gruppofrattura.it/ocs/index.php/esis/CP2006/paper/viewFile/9435/6088.

2. Roylance, D. 2001. Introduction to Fracture Mechanics, Department of Materials Science and Engineering, Massachusetts Institute of Technology, Cambridge, June 14, p. 11.

https://pdf4pro.com/view/introduction-to-fracture-mechanics-mit-38e6a9.html.

3. Felix, H.; Kim.; Shawn, P.; Moylan, Edward, J.; Garboczi. 2019. Preparation of cylindrical tensile specimens for simultaneous mechanical testing and X-xay computed tomography. https://doi.org/10.6028/NIST.IR.8234.

4. Shlyannikov, V.; Yarullin, R.; Ishtyryakov, I. 2015. Surface crack growth in cylindrical hollow specimen subject to tension and torsion, Frattura Ed Integrità Strutturale, Jun 19, vol. 9. https://doi: 10.3221/IGF-ESIS.33.37.

5. Russell, Alexander.; et al. 2020. Deformation and breakage of biofuel wood pellets, Chemical Engineering Research and Design 153: 419-426. https://doi.org/10.1016/j.cherd.2019.10.034.

6. Pysz, A.; Maj, M.; Czekaj, E. 2014. High-strength aluminium alloys and their use in foundry industry of nickel superalloys, Archives of Foundry Engineering 14(3): 71-76.

https://journals.pan.pl/dlibra/publication/102176/edition/88192/content.

7. Hussain, F.; Abdullah, S.; Nuawi, M. Z. 2016. Effect of temperature on fatigue life behaviour of aluminium alloy AA6061 using analytical approach, Journal of Mechanical Engineering and Sciences 10(3): 2324-2335. https://doi.org/10.15282/jmes.10.3.2016.10.0216.

8. Russell, A.; et al. 2018. Mechanics of Pharmaceutical Pellets - Constitutive Properties, Deformation, and Breakage Behavior, Journal of Pharmaceutical Sciences 107(2): 571-586. https://doi.org/10.1016/j.xphs.2017.08.022

9. Fatemi, Ali.; et al. 2014. Fatigue crack growth behaviour of tubular aluminium specimens with a circular hole under axial and torsion loadings, Engineering Fracture Mechanics 123: 137-147.

http://dx.doi.org/10.1016\%2Fj.engfracmech.2014.04.10.

10. Li, Ran; Hyde, Thomas, H.; Sun, Wei. 2013. Finite element prediction of fatigue crack growth in super $\mathrm{cmv}$ hollow shafts with transverse holes under combined torsional and axial loading, The Journal of Strain Analysis for Engineering Design 48(7): 457-469.

http://dx.doi.org/10.1177/0309324713492318.

11. Derpenski, L. 2019. Ductile fracture behavior of notched aluminum alloy specimens under complex nonproportional load, Materials 12(10): 1598.

https://doi.org/10.3390/ma12101598.

12. Faridmehr.; Iman.; et al. 2014. Correlation between engineering stress-strain and true stress-strain curve, American Journal of Civil Engineering and Architecture 2(21): 53-59.

http://dx.doi.org/10.12691/ajcea-2-1-6.

\section{Solomon, E. Narvydas, G. Dundulis}

\section{STRESS-STRAIN STATE ANALYSIS AND FATIGUE PREDICTION OF D16T ALLOY IN THE STRESS CONCENTRATION ZONE UNDER COMBINED TENSION-TORSION LOAD}

\section{S u m m a r y}

Engineering components undergo structural failure in their lifetime due to repetitive loads and therefore cause imbalance in their assembly and compromise their further process. Hence identifying the failure prone areas and predicting the failure modes are of significant importance in order to reduce the risk of damage and improve their working lifetime. An experimental approach was implemented and the data was attempted against finite element method-based numerical simulations. An experimental procedure was carried out on D16T Al-alloy specimens to study their mechanical behaviour and to determine their physical characteristics. The mechanical properties obtained from the experimental study are then attempted against apparent finite element modelling techniques and the material's constitutive behaviour are presented for the tension, torsion and combined tension-torsion loads. The various stress-strain state results of the simulations were used in determining the fatigue predictions and the influence of the presence of holes in the engineering components are demonstrated.

Keywords: stress concentration, finite element method, fatigue, tension-torsion load.

Received March 02, 2021

Accepted October 04, 2021 